\title{
Intractable hypoglycemia in pregnancy
}

\section{Kavita Gonsalves $^{1 *}$, Mallikarjun V. J. ${ }^{2}$, Annamma Thomas ${ }^{1}$, Ganapathi Bantwal ${ }^{2}$}

\author{
${ }^{1}$ Department of Obstetrics and Gynecology, St. John's Medical College, Bangalore, Karnataka, India \\ ${ }^{2}$ Department of Endocrinology, St. John's Medical College, Bangalore, Karnataka, India
}

Received: 30 January 2017

Accepted: 02 March 2017

\author{
*Correspondence: \\ Dr. Kavita Gonsalves, \\ E-mail: kavita12@gmail.com
}

Copyright: (C) the author(s), publisher and licensee Medip Academy. This is an open-access article distributed under the terms of the Creative Commons Attribution Non-Commercial License, which permits unrestricted non-commercial use, distribution, and reproduction in any medium, provided the original work is properly cited.

\begin{abstract}
Insulinomas are rare tumours developed from pancreatic beta cells; 27 cases of insulinoma associated with pregnancy have been reported till date, mostly diagnosed before the $16^{\text {th }}$ week. Triad of insulinoma; hypoglycemic symptoms, plasma glucose $<3.0 \mathrm{mmol} / \mathrm{L}$, symptomatic relief with glucose administration. 28-year-old primi presented to us at 29 weeks with high BP and neuroglycopenic symptoms. Repeated episodes of loss of consciousness with hypoglycemia since 14 weeks. On evaluation diagnosed to have insulinoma and managed conservatively with dietary modifications, and medical treatment. She failed to respond and pregnancy was terminated due to high BP, hypoglycemia and Doppler changes. Delivered a stillborn girl at 31 weeks. Underwent laparotomy post-delivery for enucleation of the lesion. Quick normalization of sugar levels and overall recovery remarkable. Patient is on regular follow up. Insulinoma is a rare cause of severe repeated episodes of hypoglycemia early in pregnancy.
\end{abstract}

Keywords: Hypoglycemia, Insulinoma

\section{INTRODUCTION}

We present a 28 year old primi who presented with neuroglycopenic symptoms in the early second trimester, was diagnosed empirically as insulinoma only in the third trimester and managed. With advancing pregnancy her symptoms worsened and she developed preeclampsia and had to undergo termination of pregnancy. This case highlights the diagnostic and management dilemmas of hypoglycemia in pregnancy. Insulinomas are rare endocrine tumors developed from pancreatic beta cells. First described by Harris in 1924 and 3 years later the association between insulin secreting pancreatic endocrine tumors and hypoglycemia was reported by Wilder et al. ${ }^{1}$

The incidence is 2 to 4 patients per million population per year. ${ }^{1,2}$ About 27 cases of insulinoma associated with pregnancy have been reported to date, mostly diagnosed before the 16th week. ${ }^{3}$ More than $80 \%$ are solitary benign tumors and small $(<2 \mathrm{~cm})$, often difficult to localize, have an indolent course and 5\% may be malignant with liver and lymph node metastasis. ${ }^{1,2}$ The etiology is unknown. Characteristically patients with insulinoma present with profound and symptomatic hypoglycaemia. Hypoglycemic episodes present as neurological symptoms or autonomic manifestations. Whipple and Franz described a triad unique to insulinoma; symptoms of hypoglycemia, plasma glucose $<3.0 \mathrm{mmol} / \mathrm{L}(45 \mathrm{mg} / \mathrm{dL})$, and relief of symptoms with glucose administration. ${ }^{2}$

Diagnostic criteria include: Inappropriately high levels of insulin ( $>3 \mathrm{mIU} / \mathrm{L}$ ), C-peptide ( $>0.6 \mathrm{ng} / \mathrm{mL}$ ), proinsulin ( $>5 \mathrm{pmol} / \mathrm{L}$ ), no detectable circulating oral hypoglycemic agents, during a fast test, beta-hydroxy-butyrate levels of $2.7 \mathrm{mmol} / \mathrm{L}$ or less and an increase in plasma glucose of at least $0.25 \mathrm{~g} / \mathrm{L}$ after intravenous glucagon indicate mediation of hypoglycemia by insulin. ${ }^{1,2}$ 


\section{CASE REPORT}

A 28 year primi at 29 weeks gestation, presented to us with high blood pressure and repeated episodes of loss of consciousness since one week. She gave a history of repeated episodes of loss of consciousness with seizure like activity, starting for the very first time at 14 weeks of gestation. On evaluation at 14 weeks, she was hypoglycemic with blood glucose of $26 \mathrm{mg} / \mathrm{dL}$ and responded to IV dextrose injections. She improved symptomatically. Again at 16 weeks she had repeated episodes of loss of consciousness with occasional urinary incontinence especially in the early hours of the day. MRI done was suggestive of corticovenous thrombosis (CVT) of left transverse and sigmoid sinuses.

Anticonvulsant and low molecular weight heparin was started. Her symptoms reduced but never completely subsided. Again at 29 weeks, she had similar complaints with high blood pressures and proteinuria for which she presented at our institute and was admitted. There was no other comorbid illness. She denied history of any prolonged drug intake. On examination she was obese with a prepregnancy BMI of $24 \mathrm{~kg} / \mathrm{m}^{2}$ and a BP of $140 / 100$ $\mathrm{mmHg}$. No significant findings on systemic examination. On abdominal examination uterus was corresponding to 26 weeks with FHR 146 bpm.

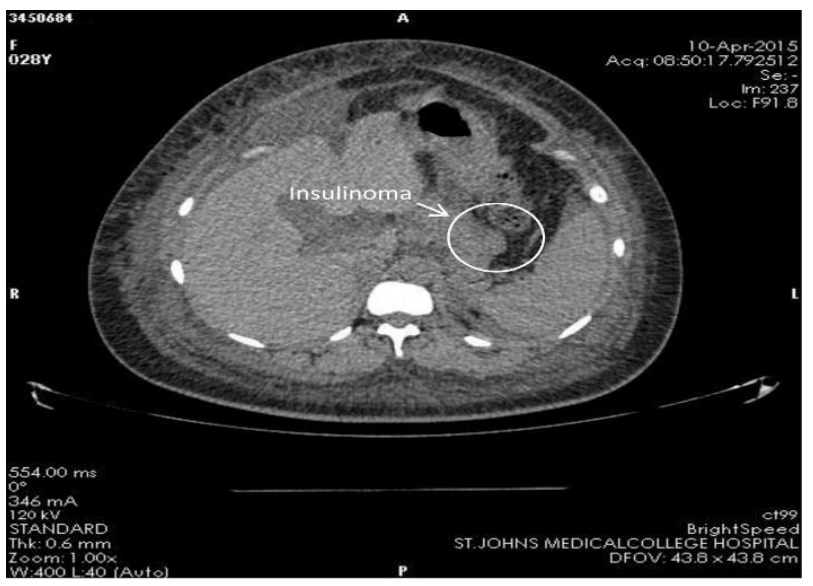

Figure 1: CT scan showing exophytic lesion in the anterior border of pancreas.

After consulting endocrinologist, she was evaluated for cause of hypoglycemia and reports were suggestive of hyperinsulinemic hypoglycemia (RBS $28 \mathrm{mg} / \mathrm{dl}$, Serum insulin-42.51 miu/L, C-peptide- $2.79 \mathrm{ng} / \mathrm{ml}$, Serum cortisol- $18.78 \mathrm{ug} / \mathrm{dl}$ ). Insulinoma was suspected and patient was subjected to further evaluation to localize the tumor. CT abdomen (Figure 1) and Endoscopic ultrasound (Figure 2) was done. She was diagnosed to have insulinoma and advised dietary modifications in the form of frequent small complex carbohydrate meals. In consult with a neurologist the previous MRI films were reviewed and repeat MRI performed both of which suggested that the patient had no CVT. Hence the anticonvulsants and LMWH were discontinued.

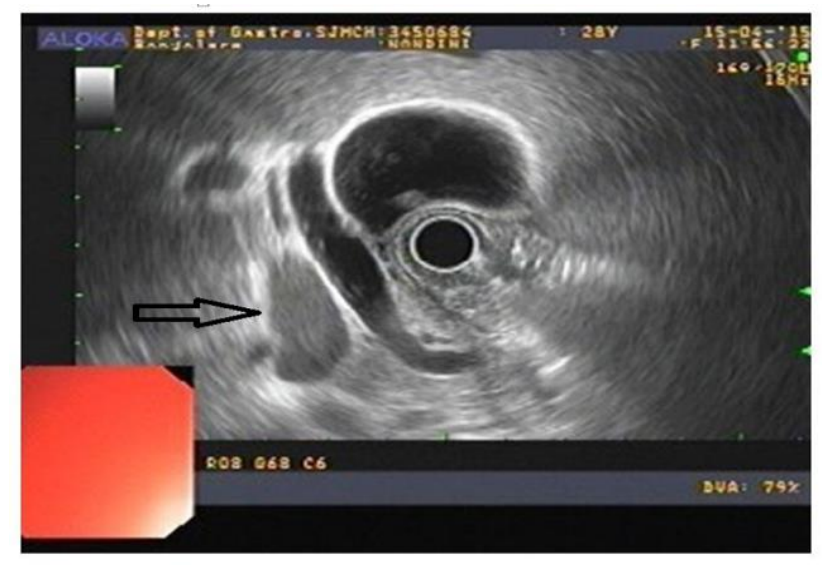

Figure 2: Single hypoechoic relatively avascular lesion $1.9 \times 0.9 \mathrm{~cm}$ seen in the body of pancreas near the surface.

The sugar levels and blood pressure were closely monitored. Patient had repeated episodes of hypoglycemia with blood glucose as low as $25-30 \mathrm{mg} / \mathrm{dL}$ despite dietary modifications. Tablet Diazoxide a category $\mathrm{C}$ drug was started in view of uncontrolled hypoglycemic episodes after discussing with the patient and her family. Dose was further escalated with no improvement. Steroid injections and injection octreotide was initiated as patient continued to have repeated hypoglycemic episodes. BP was fluctuating and growth restriction had set in. Obstetric Doppler was suggestive of brain sparing effect.

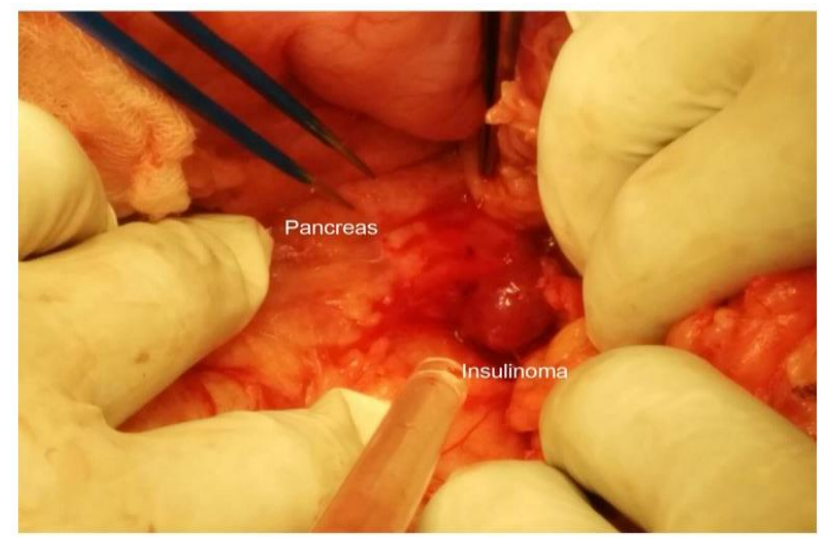

Figure 3: Intraoperative finding of a swelling at the junction of the body and tail of pancreas.

She poorly responded to the dietary modifications and medical management. Hence a collaborative decision to terminate pregnancy was taken in order to prevent hypoxic neurological damage both to the mother and fetus. She was induced and delivered a stillborn girl baby of $960 \mathrm{~g}$ at 31 weeks gestation. Immediate postpartum the hypoglycemic episodes continued, and she underwent laparotomy with enucleation of the lesion. Intraoperatively (Figure 3) a $1.5 \times 0.8 \mathrm{~cm}$ firm swelling at the junction of the body and tail of pancreas anteriorly was identified and enucleated after confirming the lesion with intraoperative ultrasound. 
Postoperatively patient showed quick normalization of her sugar levels and her overall recovery was satisfactory. Patient was discharged on postoperative day 5. Histopathology report confirmed the diagnosis of insulinoma. Patient is on regular follow up and is stable.

\section{DISCUSSION}

Insulinoma is a rare tumor of the pancreatic islet beta cells, can occur coincidentally with pregnancy or postpartum period. Real incidence of this tumor is probably underestimated owing to difficulties in reaching diagnosis in such periods. ${ }^{4}$ Evidence based management is very limited hence decisions on investigation and therapy must be individualized and undertaken jointly by the multidisciplinary medical team and the patient. ${ }^{3,5}$ The optimal strategy will depend on the nature and stage of the endocrine tumor, gestational age, treatments available and patient's wishes. There is good evidence that insulin secretion increases rapidly from the beginning of pregnancy because of beta cell proliferation and enhanced beta cell sensitivity to glucose stimulus as a result of hormonal changes. ${ }^{2}$ Studies have suggested that insulin sensitivity is enhanced during early pregnancy and this explains why insulinoma occurs early during pregnancy.

Most cases of insulinoma manifest during the first trimester. Second and third trimesters of pregnancy are known to be an insulin resistant state due to placental counter-regulatory hormones like human placental lactogen, estrogen, progesterone, and increase in free cortisol levels. ${ }^{1,2}$ These factors may have contributed to masking disease progression in later stages of pregnancy and patient presenting postpartum. Insulinomas are generally thought to be difficult to localize due to their small size. ${ }^{2}$ Intraoperative palpation and ultrasound examination of the pancreas had been thought to be the best methods to detect insulinomas. CT-scan is the first investigation performed to localize insulinomas.

Most insulinomas are small, isodense with the pancreas on pre-contrast images, then hypervascular on arterial phase images, but sometimes are more easily detected on portal venous phase images. EUS is preoperative imaging study of choice and can identify tumors as small as 2 to $3 \mathrm{~cm}$ with sensitivity of 70 to $90 \%$, and $100 \%$ specificity. ${ }^{1}$ Surgery remains the only curative treatment of insulinomas. Long-term remission can be achieved by surgery in $95 \%$ of patients according to a recent study. ${ }^{2}$ Two different types of surgery can be performed: minimal resection i.e. either tumor enucleation whenever it is possible or central pancreatectomy, or a more extended resection, i.e. left-sided pancreatectomy or pancreaticoduodenectomy.

Frequent meals and intravenous glucose infusions are adequate for hypoglycemic control but generally not sufficient to avoid hypoglycaemia., ${ }^{2,6}$ Diazoxide (benzothiazide) allows direct suppression of insulin secretion by beta cells through its effect on K-ATP channel. It stimulates hepatic gluconeogenesis and lowers glucose utilization by muscle cells. Somatostatin analogues can achieve normalization of plasma glucose levels in $50-60 \%$ of the patients.

Review of literature shows that most of the cases with hypoglycemia in pregnancy presented around 16 weeks gestation. ${ }^{4,6}$ Only one patient became symptomatic at 35 weeks gestation (malignant insulinoma). Symptoms developed when insulin sensitivity rebounded after delivery in the remaining cases. Dietary modifications in the form of frequent meals and intravenous glucose were enough to control hypoglycemia in majority of cases. Five patients were operated at $12-17$ weeks. ${ }^{4,6}$

In our case because of the rarity of insulinoma the diagnosis was delayed. Though review of literature points towards improvement of symptoms in second and third trimester, in our case symptoms worsened as diagnosis and management was delayed. Once diagnosed patient failed to respond to different modalities of management which was unusual.

Our case highlights the need for high index of suspicion of insulinoma, though a rare cause in patients with severe repeated episodes of hypoglycemia early in pregnancy. If the diagnosis was appropriate then we could have managed her with a conservative approach until delivery. Nextly in insulinoma the investigative modalities may not always be conclusive. If diagnosis is confirmed during gestation surgery can be performed in the second trimester. In a low resource setting like ours it is very important to rely on our clinical acumen and such a possibility has to be taken into account in the differential diagnosis of symptomatic hypoglycemia early in pregnancy. Misdiagnosis has been fatal. Careful management during pregnancy and aggressive treatment after delivery are essential.

\section{Funding: No funding sources \\ Conflict of interest: None declared \\ Ethical approval: Not Required}

\section{REFERENCES}

1. Melmed S, Polonsky SK, Larsen RP, Kronerberg MH. Williams Text Book of Endocrinology, $12^{\text {th }}$ Edition. Elsevier Saunders; 2011.

2. Chakradhar K, Sisodiya RR, Prabhu M, Maru R. A rare presentation of insulinoma as postpartum hypoglycaemia. World J Surg Res. 2014;3:1-5.

3. Rodrigues Queiroz AJ, Nazareno LS, Miranda JE, de Azevedo AE, Teixeira da Cruz CA, Pirani Carneiro F et al. Insulinoma diagnosed in the postpartum: clinical and immunohistochemical features. Gynecol Endocrinol. 2012;28(8):633-6.

4. Lansdown A, Rees DA. Endocrine oncology in pregnancy. Best Pract Res Clin Endocrine Metab. 2011;25(6):911-26.

5. Vezzosi D, Bennet A, Maiza JC, Buffet A, Grunenwald S, Fauvel J et al. Diagnosis and treatment 
of insulinomas in the adults. In Basic and Clinical Endocrinology Up-to-Date. book edited by Fulya Akin. Intech Publisher; 2011:135-176.

6. Diaz AG, Herrera J, López M, Félix M. Puchulu, Pedro Ferraina, Oscar D. Bruno. Insulinoma associated with pregnancy. Fertil Steril. 2008;90(1):199e1-4.
Cite this article as: Gonsalves K, Mallikarjun VJ, Thomas A, Bantwal G. Intractable hypoglycemia in pregnancy. Int J Reprod Contracept Obstet Gynecol 2017;6:1672-5. 